\title{
Human ChIR1 Stimulates Endonuclease Activity of hFen1 Independently of ATPase Activity
}

\author{
Do-Hyung Kim, Jeong-Hoon Kim, ${ }^{\dagger}$ Byoung Chul Park, ${ }^{\dagger}$ Do Hee Lee, ${ }^{\ddagger}$ Sayeon Cho, ${ }^{\S, *}$ and Sung Goo Park ${ }^{\dagger}, *$ \\ Bio Research Team, LG Household \& Health Care Research Park, Daejeon 305-343, Korea \\ ${ }^{\dagger}$ Medical Proteomics Research Center, Korea Research Institute of Bioscience \& Biotechnology, Daejeon 305-806, Korea \\ *E-mail: sgpark@kribb.re.kr \\ ${ }^{\star D}$ Department of Biotechnology, College of Nature Sciences, Seoul Women's University, Seoul 139-774, Korea \\ ${ }^{\S}$ College of Pharmacy, Chung-Ang University, Seoul 156-756, Korea. ${ }^{*}$ E-mail: sycho@cau.ac.kr \\ Received June 11, 2014, Accepted June 18, 2014
}

\begin{abstract}
Human ChlR1 protein (hChlR1), a member of the cohesion establishment factor family, plays an important role in the segregation of sister chromatids for maintenance of genome integrity. We previously reported that hChlR1 interacts with hFen 1 and stimulates its nuclease activity on the flap-structured DNA substrate covered with RPA. To elucidate the relationship between hChlR1 and Okazaki fragment processing, the effect of hChlR1 on in vitro nuclease activities of hFen1 and hDna2 was examined. Independent of ATPase activity, hChlR1 stimulated endonuclease activity of hFen1 but not that of hDna2. Our findings suggest that the acceleration of Okazaki fragment processing near cohesions may aid in reducing the size of the replication machinery, thereby facilitating its entry through the cohesin ring.
\end{abstract}

Key Words : ChlR1, Dna2, Fen1, Okazaki fragment processing

\section{Introduction}

In eukaryotic cells, DNA replication is initiated at multiple origins and proceeds bidirectionally from each origin to flanking DNA. For simultaneous synthesis of both strands, the leading strand is continuously replicated by DNA polymerase $\varepsilon$ whereas the lagging strand is synthesized as short Okazaki fragments by primase, DNA polymerases $\alpha$ and $\delta$, and then ligated into one long DNA strand. These enzymes build up the replisome in conjunction with other components, such as Mcm2-7 helicase, PCNA, and RFC. ${ }^{1-4}$ In yeast, Okazaki fragments are processed by well-organized multiple enzymatic reactions involving Dan2, Fen1 and DNA ligase I, and subsequently ligated into a long DNA strand. ${ }^{5,6}$ To complete synthesis of lagging strand DNA, Okazaki fragments must be processed about 50 million times per cell cycle in mammalian cells. Failure of Okazaki fragment processing in yeast is associated with increasing DNA double-strand breaks, higher mutation rates and generation of genome instabilities. ${ }^{7,8}$ Efficient processing of the Okazaki fragment is therefore essential for DNA replication and cell cycle progression. Human Fen1 protein (hFen1) can cleave the flap structure at the junction between ssDNA and dsDNA, an intermediate formed during Okazaki fragment processing, resulting in ligatable nicked dsDNA. This step is crucial for cells to maintain genome integrity. Two sister chromosomes replicated during $\mathrm{S}$ phase are linked by the cohesion complex until metaphase and separated into daughter cells during anaphase. Human ChlR1 protein (hChlR1) is an ATP-dependent DNA helicase interacting with hFen1. Depletion of hChlR1 in HeLa cells leads to abnormal separation of sister chromatids. ${ }^{9}$
In this study, to clarify the relationship between Okazaki fragment processing and hChlR1, we examined the effect of hChlR1 on in vitro endonuclease activity of hFen 1 . We present the evidence that hChlRlinduces acceleration of Okazaki fragment processing near cohesion, resulting from stimulation of hFen1 activity to reduce the size of replisome structure.

\section{Experimental Section}

Oligonucleotides, Nucleoside Triphosphates and Enzymes. The oligonucleotides used to construct DNA substrates (listed in Table 1) were commercially synthesized (Genotech, Daejeon, Korea) and gel-purified prior to use. Nucleoside triphosphates were obtained from Boehringer Mannheim and $\left[\gamma-{ }^{32} \mathrm{P}\right]$ ATP $(>3000 \mathrm{Ci} / \mathrm{mmol})$ was purchased from Amersham Pharmacia Biotech. Enzymes used in this study including restriction endonucleases, T4 DNA ligase and T4 polynucleotide kinase were purchased from Enzynomics (Daejeon, Korea).

Preparation of Substrates. Oligonucleotide-based partial duplex substrates, except for the equilibrating flap substrates, were prepared as previously described ${ }^{10}$ using the synthetic oligonucleotides listed in Table 1. Equilibrating flap substrates were prepared as described previously. ${ }^{11}$ Briefly, a upstream oligonucleotide was labeled at 5 -end with $[\gamma$ $\left.{ }^{32} \mathrm{P}\right] \mathrm{ATP}$ and T4 polynucleotide kinase. The 5'-labeled oligonucleotide was annealed to a template oligonucleotide and a downstream oligonucleotide (molar ratio of 1:3:10, respectively). The annealed substrates were purified by polyacrylamide gel electrophoresis ${ }^{10}$ and their specific activities $(2,500$ $\mathrm{cpm} / \mathrm{fmol}$ ) were determined by liquid scintillation counting 
Table 1. Oligonucleotides used in this study

\begin{tabular}{ccl}
\hline Primer & Size $(\mathrm{nt})$ & \multicolumn{1}{c}{ Sequence } \\
\hline $\mathrm{D}_{\mathrm{F}}$ & 40 & 5'-AGG TCT CGA CTA CCA CCC GTC CAC CCG ACG CCA CCT CCT G-3' $^{\prime}$ \\
$\mathrm{T}_{\mathrm{F}}$ & 51 & 3'-GCT GGC ACG GTC GGA TTT AAA GTT AGG GCA GGT GGG CTG CGG TGG AGG ACG-5' \\
$\mathrm{U}_{\mathrm{F}}$ & 26 & 5'-CGA CCG TGC CAG CCT AAA TTT CAA TC-3' \\
$\mathrm{D}_{\mathrm{E}}$ & 58 & 5'-TAG GTC TCG ACG ACT AAC TCT AGT CGT TGT TCC ACC CGT CCA CCC GAC GCC ACC TCC TG-3' \\
$\mathrm{T}_{\mathrm{E}}$ & 85 & 3'-GCT GGC ACG GTC GGA TTT AAA GTT ATC CAG AGC TGC TGA TTG AGA TCA GCA ACA AGG TGG \\
& & GCA GGT GGG CTG CGG TGG AGG ACG-5' \\
$\mathrm{U}_{\mathrm{E}}$ & 39 & 5'-CGA CCG TGC CAG CCT AAA TTT CAA TAG GTC TCG ACG ACT-3' \\
729 & 52 & 5'-CGA ACA ATT CAG CGG CTT TAA CCG GAC GCT CGA CGC CAT TAA TAA TGT TTT C-3' \\
$5 \mathrm{TY}-1$ & 50 & 3'-GGC AAT CGT CAA GCG GAA CAC GGA TCG AGC TGC GGT AAT TAT TAC AAA AG-5' \\
$5 \mathrm{~TB}$ & 25 & 5'-CCG TTA GCA GTT CGC CTT GTG CCT A-3' \\
$5 \mathrm{TBG}$ & 26 & 5'-CCG TTA GCA GTT CGC CTT GTG CCT AG-3' \\
\hline
\end{tabular}

\section{(Beckman).}

Purification of Human Fen1 and Other Proteins. The pET-23d(+)-hFen1 plasmid was constructed as described previously. ${ }^{12,13}$ The plasmid carrying human Fen1 with Cterminal $6 \times$ His-tag was introduced into E. coli BL21 (DE3) CodonPlus-RIL (Stratagene) and the recombinant protein was induced at $\mathrm{A}_{600}=0.4$ by adding $0.4 \mathrm{mM}$ isopropyl $\beta$-Dthiogalactopyranoside (IPTG) at $30^{\circ} \mathrm{C}$ for $3 \mathrm{~h}$. Human Fen1 was purified according to the previous procedure. ${ }^{12}$ Peak fractions from Mono S column chromatography were subjected to glycerol gradient sedimentation, as described elsewhere. ${ }^{14}$ Active peak fractions from the glycerol gradient were pooled, aliquotted, and stored at $-80^{\circ} \mathrm{C}$ until further use. hDna2 protein waskindly provided by Dr. Jerard Hurwitz.

Assay of Nuclease Activity. To measure the endonuclease activities of $\mathrm{hDna} 2$ and $\mathrm{hFen} 1$, the reaction mixture $(20 \mu \mathrm{L})$ containing $50 \mathrm{mM}$ Tris- $\mathrm{HCl}(\mathrm{pH} 7.8), 50 \mathrm{mM} \mathrm{NaCl}, 8 \mathrm{mM}$ $\mathrm{MgCl}_{2}, 2 \mathrm{mM}$ DTT, $0.25 \mathrm{mg} / \mathrm{mL}$ BSA and 5'-[ $\left.{ }^{32} \mathrm{P}\right]$-labeled DNA substrate ( $15 \mathrm{fmol}$ ) was used. Enzymes were diluted to the appropriate concentrations prior to use in buffer containing $50 \mathrm{mM}$ Tris- $\mathrm{HCl}(\mathrm{pH} 7.8), 2 \mathrm{mM} \mathrm{DTT}, 0.5 \mathrm{mg} / \mathrm{mL}$ BSA, $10 \%$ glycerol, $0.5 \mathrm{M} \mathrm{NaCl}$ and $0.02 \%$ Nonidet P-40. After standing on ice for $5 \mathrm{~min}$, reaction mixtures were incubated with enzymes at $37^{\circ} \mathrm{C}$ for $10 \mathrm{~min}$ and reaction was then terminated by adding $6 \times$ stop solution $(60 \mathrm{mM}$ EDTA, $\mathrm{pH} 8.0,40 \%$ (w/v) sucrose, $0.6 \%$ SDS, $0.25 \%$ bromophenol blue and $0.25 \%$ xylene cyanol). Cleavage products were resolved on a $10 \%$ polyacrylamide gel containing $0.1 \%$ SDS in $0.5 \mathrm{X}$ TBE (electrophoresis at $150 \mathrm{~V}$ for $40 \mathrm{~min}$ ) or a $20 \%$ sequencing gel for the higher resolution. Gels were dried on DEAE-cellulose paper and subjected to autoradiography. The amount of product formed was quantitated using a PhosphorImager.

\section{Results and Discussion}

hChIR1 Stimulates Nuclease Activity of hFen1 with Various Substrates. In a previous study, we reported that hChlR1 stimulates hFen 1 nuclease activity in a reaction using the fixed-flap structured DNA substrate covered with RPA. To compare the stimulation of hFen 1 activity by
hChlR1 on substrates with different structure, we used fixedflap and equilibrating-flap structured substrates. As shown in Figure 1(a), hChlR1 could stimulate nuclease activity of hFen 1 on both the equilibrating-flap structured and fixedflap structured substrates. However, hFen 1 exhibited slightly

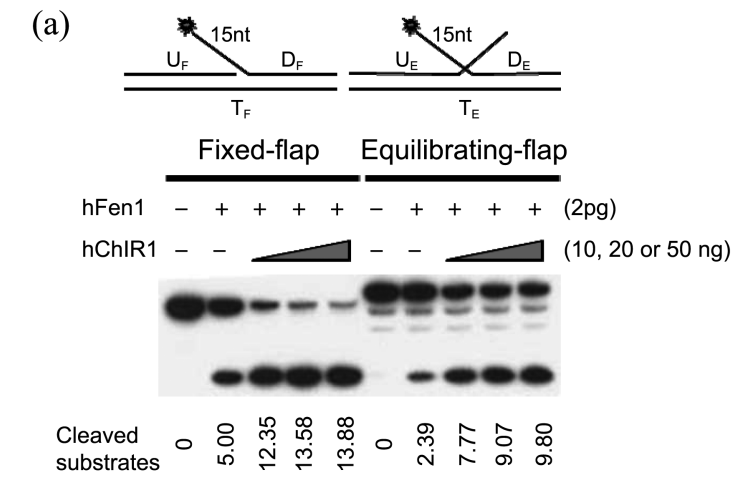

(b)
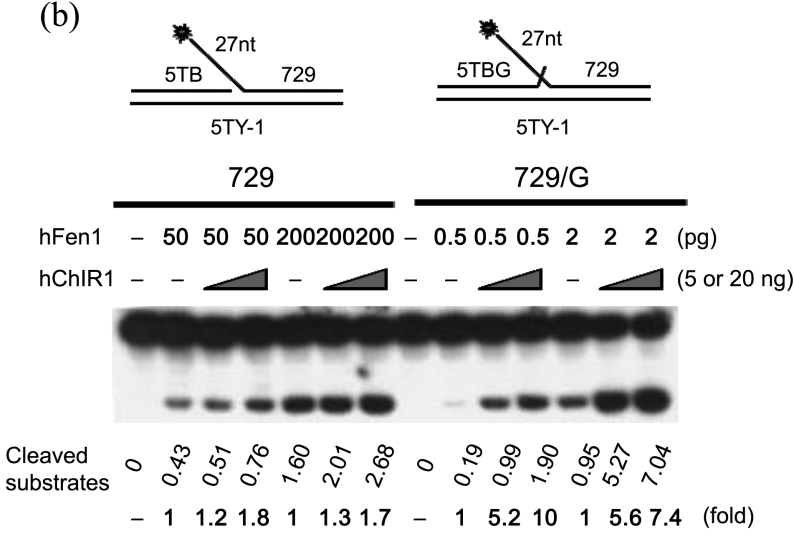

Figure 1. hChlR1 stimulates the cleavage of various substrates by hFen1. (a) Endonuclease activity of hFen 1 was measured with the indicated amounts of hFen1 and hChlR1 as described in Experimental Section. The fixed-flap and equilibrating-flap substrates, whose structures are shown at the top of the gels, were prepared as described. Reaction products were separated on a $10 \%$ polyacrylamide gel and quantified. The amount of cleavage product is indicated at the bottom of the gels. The asterisk in the substrate indicates the position of the radioactive label. (b) Similar to Figure 1(a), 729 and $729 / \mathrm{G}$ substrates were used to assay the effects of hChlR1 on the endonuclease activity of hFen1. 
weaker activity with the equilibrating-flap structured substrate and cleaved only up to $65 \%$ of total substrate since only the 5'-overhang flap-structured DNA could be cleaved. To further examine the stimulatory effect of hChlR1, we then employed substrates containing a long flap that easily generate secondary structures (Fig. 1(b)). Up-regulation of hFen 1 activity by hChlR 1 resulted in removal of the long flap from the 729/G substrate containing a 1nt 3'-overhang. Stimulation of hFen 1 activity by hChlR1 on substrate 729 was lower than that on substrate $729 / \mathrm{G}$ since much higher amount of hFen1 (as much as 100 times) was required to generate similar amounts of cleaved products from substrate
729. These results clearly demonstrate that hChlR1 stimulates the endonuclease activity of hFen 1 on substrates with equilibrating-flap or fixed-flap structures.

ATPase Activity of hChIR1 and hPCNA is Dispensable for Stimulation of hFen1 Activity. hChlR1, a member of the DEAD/DEAH family of helicases, translocates singlestranded DNA in $5^{\prime}$ to $3^{\prime}$ direction in the presence of ATP. ${ }^{9}$ To determine whether ATPase activity is required for stimulation of $\mathrm{hFen} 1$ by $\mathrm{hChlR} 1, \mathrm{hFen} 1$ nuclease activity was measured using the wild-type protein and the helicase activity-dead mutant referred as hChlR1 (KR). Similar to the wild-type protein, hChlR1 (KR) mutant also stimulated
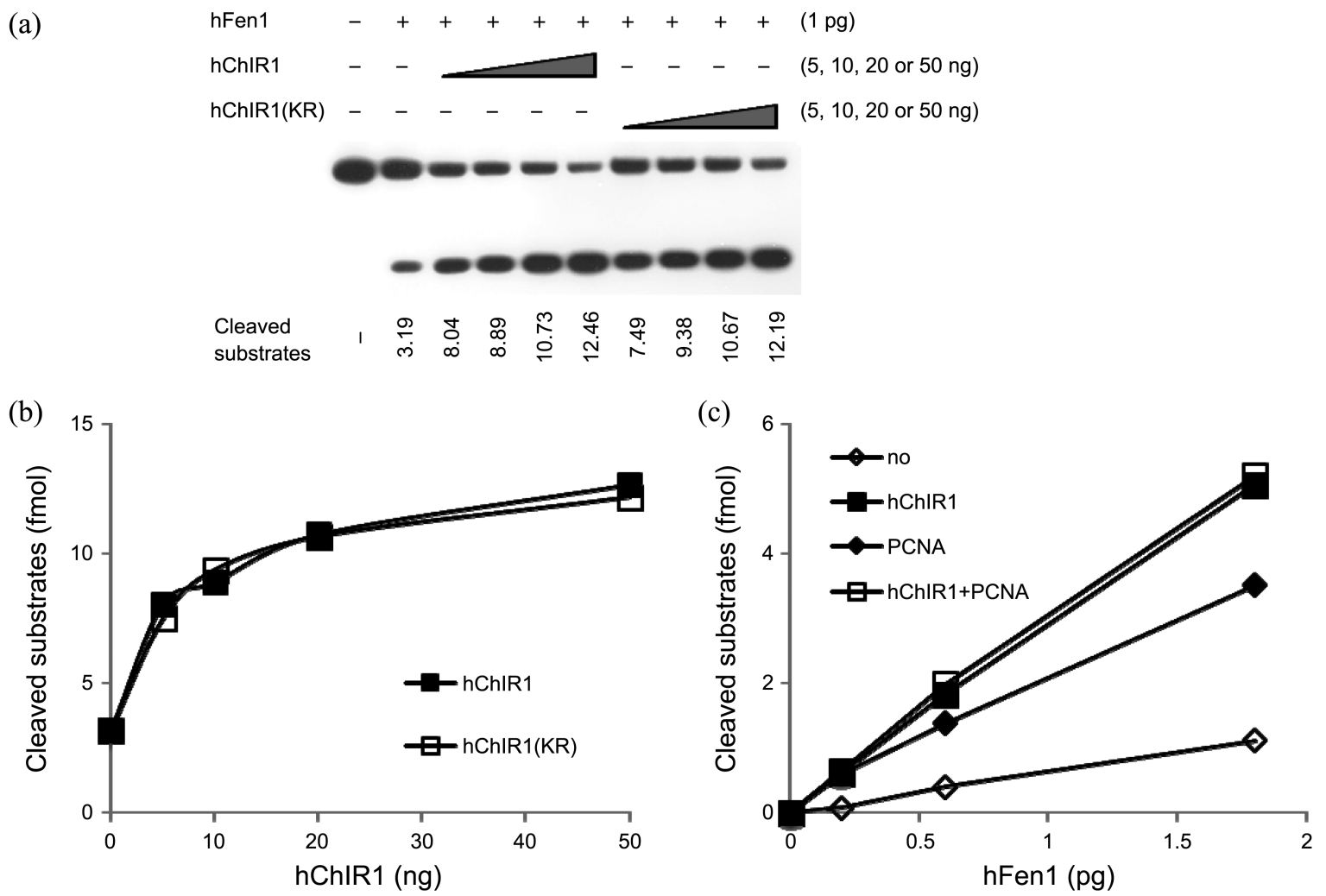

(d)

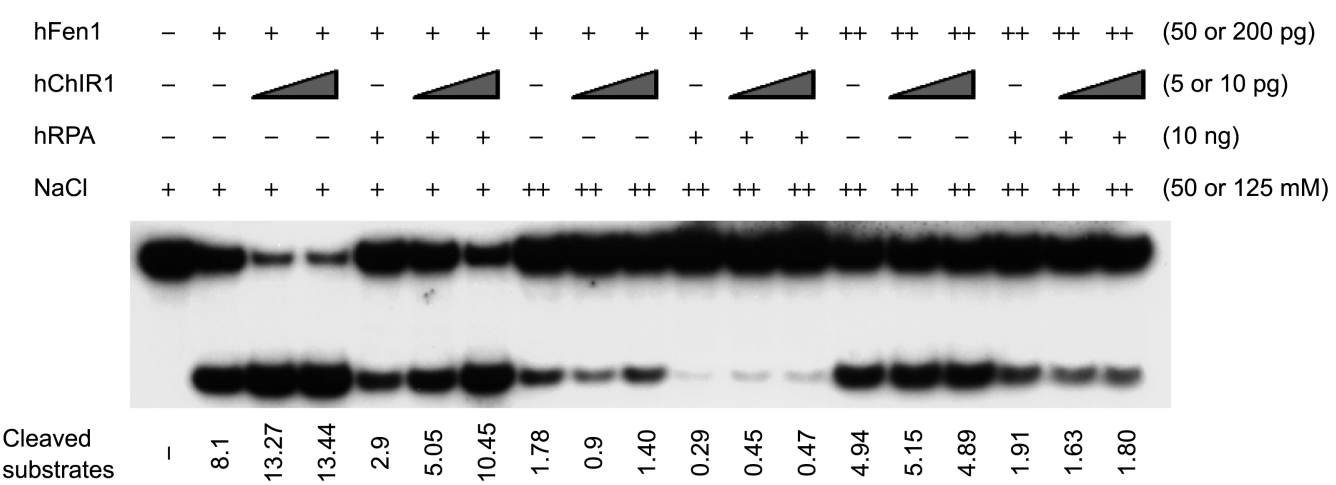

Figure 2. ATPase activity and PCNA are dispensable for hFen1 activation by hChlR1. (a) Endonuclease activity of hFen 1 was measured in the presence of hChIR1 (the wild-type) or hChIR1(KR) (ATPase activity-deficient mutant) using the fixed flap substrate. The amounts of added hChlR1 are indicated at the right of the gel. (b) Graph of the data obtained in Figure 2(a). (c) Effects of hFen1 on stimulating hChlR1 and hPCNA were examined using the $729 / \mathrm{G}$ substrate in the presence of increasing amounts of hFen1. (d) Influence of salt concentration on $\mathrm{hFen} 1$ activation by hChlR1. Endonuclease activity of hFen1 was measured under different salt concentrations (50 or $125 \mathrm{mM})$ using hRPA-covered or naked $729 / \mathrm{G}$ substrates. Due to the inhibitory effects of high salt concentrations on hFen1 activity, varying amounts of $\mathrm{hFen} 1$ were used. The amounts of cleavage products are indicated at the bottom of the gel. 


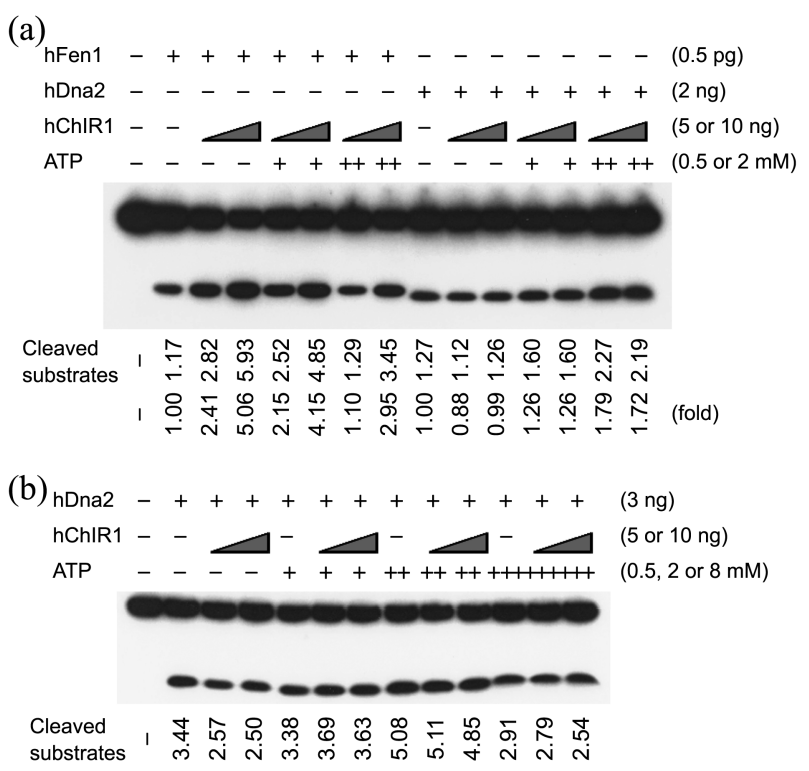

Figure 3. hChlR1 does not stimulate hDna2 activity. (a) Endonuclease activities of $\mathrm{hFen} 1$ and $\mathrm{hDna} 2$ with different concentrations of ATP were measured in the presence of hChlR1 and 729/ $\mathrm{G}$ substrate. The amounts of cleavage products and stimulation fold are indicated at the bottom of the gel. (b) With three different concentrations of ATP $(0.5,2$ or $8 \mathrm{mM})$, endonuclease activity assays of hDna2 were performed in the presence of hChlR 1 and 729 substrate.

hFen 1 activity indicating that $5^{\prime}$ to $3^{\prime}$ helicase activity of $\mathrm{hChlR} 1$ is dispensable for the stimulation of hFen 1 nuclease activity (Fig. 2(a) and 2(b)). Since hPCNA interacts with hFen1 through the PIP domain and stimulates its nuclease activity, ${ }^{15}$ we then examined whether hChlR1 and hPCNA exert a synergistic stimulatory effect on hFen1 activity. As shown in Figure 2(c), no synergistic or additive effects on hFen 1 activity were observed. Because the salt concentration is important for the enzymatic activities of $\mathrm{hFen} 1$ and hChlR1, we also performed assays under high salt conditions in the presence of hRPA. In contrast to the effects seen under low salt concentration, hChlR1 was unable to stimulate hFen 1 activity under these conditions even when the substrate was covered with RPA (Fig. 2(d)). These observations suggest that the concentration of salt is critical for stimulation of hFen1 by hChlR1.

hChIR1 Does Not Stimulate hDna2 Nuclease Activity. In yeast, processing of Okazaki fragment is carried out by Dna2 and Rad27, a yeast homolog of human Fen1, which generates ligatable nick structures of DNA. ${ }^{5}$ The activities of these enzymes are regulated by proteins involved in DNA replication and repair. For example, endonuclease activity of Dna2 is stimulated by RPA, while the activity of $\operatorname{Rad} 27$ is inhibited by RPA. ${ }^{5}$ We also examined whether hChlR 1 is capable of stimulating the endonuclease activity of hDna2 in the presence of ATP. As shown in Figure 3(a) and 3(b), hChlR1 did not affect hDna2 activity even in the presence of ATP. These findings suggest that hChlR1 stimulates the endo- nuclease activity of hFen1 specifically. In higher eukaryotes, contrary to yeast, Fen1 is more important than Dna2 during the processing of Okazaki fragment. ${ }^{16}$ It is plausible that the endonuclease activity of $\mathrm{hDna} 2$ is stimulated by ATP to a much lesser extent since this protein possesses helicase activity that aids in flap cleavage (Fig. 3(b)).

\section{Conclusions}

In this study, we found that hChlR1 cooperatively stimulates endonuclease activity of hFen1, thus allowing efficient cleavage of model substrates even if DNA is covered with RPA protein. Previously hChlR1, a cohesion establishment factor, was identified as an activator of hFen1. Here we demonstrated that the ATPase activity of hChlR1 is dispensable for stimulation of $\mathrm{hFen} 1$ activity, raising a possibility that the stimulatory effect of hChlR1 does not result from resolving the secondary structure of the flap via helicase activity. Further studies will be needed to clarify the precise relationship between hChlR1 and the machinery for lagging strand DNA synthesis.

Acknowledgments. We thank Dr. Jerard Hurwitz for providing the proteins. We are also grateful to Drs. KwangHee Bae and Sang-Chul Lee for helpful advices and careful reading of the manuscript. This work was supported by a grant from the National Research Foundation (NRF-20110028172).

\section{References}

1. Burgers, P. M. J. Biol. Chem. 2009, 284, 4041.

2. Kunkel, T. A.; Burgers, P. M. Trends Cell Biol. 2008, 18, 521.

3. McElhinny, S. A. N.; Gordenin, D. A.; Stith, C. M.; Burgers, P. M. J.; Kunkel, T. A. Molecular Cell 2008, 30, 137.

4. Pursell, Z. F.; Isoz, I.; Lundstrom, E. B.; Johansson, E.; Kunkel, T. A. Science 2007, 317, 127.

5. Bae, S. H.; Bae, K. H.; Kim, J. A.; Seo, Y. S. Nature 2001, 412, 456.

6. MacNeill, S. A. Curr. Biol. 2001, 11, R842.

7. Greene, A. L.; Snipe, J. R.; Gordenin, D. A.; Resnick, M. A. Hum. Mol. Genet. 1999, 8, 2263.

8. Sommers, C. H.; Miller, E. J.; Dujon, B.; Prakash, S.; Prakash, L. J. Biol. Chem. 1995, 270, 4193.

9. Farina, A.; Shin, J. H.; Kim, D. H.; Bermudez, V. P.; Kelman, Z.; Seo, Y. S.; Hurwitz, J. J. Biol. Chem. 2008, 283, 20925.

10. Bae, S. H.; Choi, E.; Lee, K. H.; Park, J. S.; Lee, S. H.; Seo, Y. S. J. Biol. Chem. 1998, 273, 26880.

11. Kao, H. I.; Henricksen, L. A.; Liu, Y.; Bambara, R. A. J. Biol. Chem. 2002, 277, 14379.

12. Storici, F.; Henneke, G.; Ferrari, E.; Gordenin, D. A.; Hubscher, U.; Resnick, M. A. EMBO J. 2002, 21, 5930.

13. Stucki, M.; Jonsson, Z. O.; Hubscher, U. J. Biol. Chem. 2001, 276, 7843.

14. Bae, S. H.; Seo, Y. S. J. Biol. Chem. 2000, 275, 38022.

15. Warbrick, E.; Lane, D. P.; Glover, D. M.; Cox, L. S. Oncogene 1997, 14, 2313.

16. Lee, M. H.; Hollis, S. E.; Yoo, B. H.; Nykamp, K. Biochem. Biophys. Res. Commun. 2011, 407, 495. 